\title{
An adjuvanted respiratory syncytial virus fusion protein induces protection in aged BALB/c mice
}

\author{
Anu Cherukuri ${ }^{1}$, Kate L Stokes ${ }^{2,3}$, Kathryn Patton ${ }^{1}$, Howard Kuo ${ }^{1}$, Kaori Sakamoto ${ }^{4}$, Stacie Lambert ${ }^{1}$, \\ Elizabeth Stillman', Martin L Moore ${ }^{2,3}$ and Sujin Lee ${ }^{2,3^{*}}$
}

\begin{abstract}
Background: Respiratory Syncytial Virus (RSV) causes significant disease in the elderly, in part, because immunosenescence impairs protective immune responses to infection in this population. Despite previous and current efforts, there is no RSV vaccine currently licensed in infants or elderly adults. Adjuvanted RSV subunit vaccines have the potential to boost waning immune responses and reduce the burden of RSV disease in the elderly population.
\end{abstract}

Results: We used an aged BALB/C mouse model to evaluate immune responses to RSV Fusion (F) protein in the absence and presence of an alum adjuvant. We demonstrate that aged BALB/c mice immunized with alum-adjuvanted RSV F protein had significantly reduced lung viral titers at day 4 following challenge with wild-type (wt) RSV. Serum neutralizing antibody titers measured on day 27 correlated with protection in both young and aged vaccinated mice, although the magnitude of antibody titers was lower in aged mice. Unlike young mice, in aged mice, alum-adjuvanted RSV F did not induce lung $T_{H} 2$-type cytokines or eosinophil infiltration compared to non-adjuvanted F protein following wt RSV challenge.

Conclusion: Our studies demonstrate that neutralizing anti-RSV antibody titers correlate with protection in both young and aged BALB/C mice vaccinated with RSV F protein vaccines. The $\mathrm{F}+$ alum formulation mediated greater protection compared to the non-adjuvanted $\mathrm{F}$ protein in both young and aged mice. However, while alum can boost F-specific antibody responses in aged mice, it does not completely overcome the reduced ability of a senescent immune system to respond to the RSV F antigen. Thus, our data suggest that a stronger adjuvant may be required for the prevention of RSV disease in immunosenescent populations, to achieve the appropriate balance of protective neutralizing antibodies and effective $T_{H} 1$-type cytokine response along with minimal lung immunopathology.

Keywords: Respiratory Syncytial Virus, Immunosenescence, Alum, Adjuvant, Aged mice

\section{Background}

Respiratory Syncytial Virus (RSV) is the most important viral pathogen responsible for lower respiratory tract illness in infants, and a major cause of morbidity and mortality in the elderly $[1,2]$. In elderly patients, RSV caused $11 \%$ of hospitalizations for pneumonia [1,3]. The overwhelming majority of RSV-associated deaths in the USA are in the elderly $[1,4,5]$. To date, there is no approved vaccine to prevent RSV disease and the requisite

\footnotetext{
* Correspondence: sujin.lee@emory.edu

2Department of Pediatrics, Emory Children's Center, Emory University, Atlanta, GA, USA

${ }^{3}$ Children's Healthcare of Atlanta, Atlanta, GA, USA

Full list of author information is available at the end of the article
}

components for vaccine efficacy against RSV disease in the elderly are largely unknown. Immunosenescence is a hallmark of aging and impairs the capacity to respond to vaccination, as well as the ability to prevent infection. Mechanisms of immunosenescence that result in weaker responses to vaccination in the elderly are not well understood. We undertook this study because evaluation of vaccine responses in aged mouse models may be informative in understanding vaccine-induced immune responses in the elderly.

Purified RSV fusion (F), attachment (G), and matrix (M) proteins have been developed as subunit vaccines $[6,7]$. RSV fusion $(F)$ protein has been tested in the clinic as a potential subunit vaccine [6,7], and one vaccine

\section{() Biomed Central}


(PFP-2) was tested in adults over age $60[8,9]$. However, RSV subunit vaccines have been plagued with poor immunogenicity in the elderly $[10,11]$. In addition, it has been shown that immunization with purified $\mathrm{F}$ protein enhanced pulmonary histopathology in cotton rats and mice $[12,13]$. Thus, it appears that RSV F protein alone is insufficient to show protection in aged mice due to poor immunogenicity. We hypothesized that adjuvant mixed with $\mathrm{F}$ protein may overcome poor immunogenicity, reduce histopathology, and induce protection in
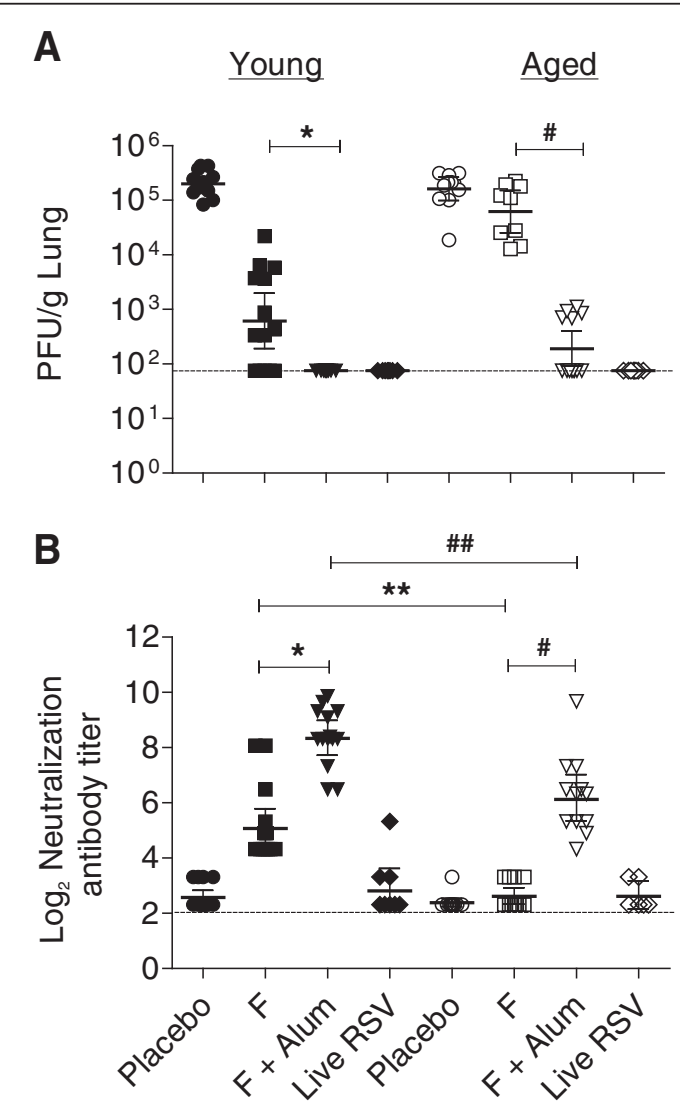

C

\section{Young}

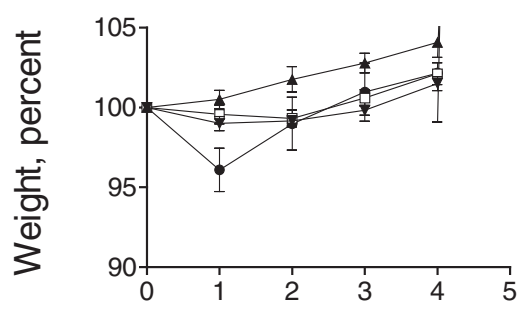

Aged

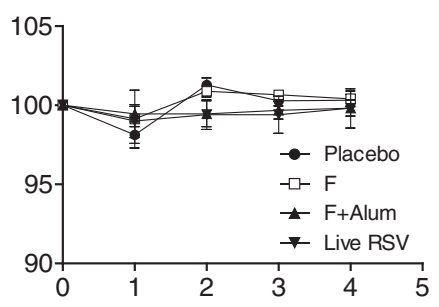

\section{Days post-challenge}

Figure 1 Lung viral loads and serum neutralizing antibodies in vaccinated young and aged BALB/c mice. Groups of mice $(n=14)$ were vaccinated twice i.m. and sera were collected by submandibular bleeding at day 27 . Mice were challenged intranasally with $10^{6}$ PFU RSV strain A2 at day 28. (A) Viral load. Lungs were harvested 4 days post-challenge, homogenized, and infectious RSV was titrated by plaque assay. ${ }^{*} P<0.05$ comparing $\mathrm{F}$ to $\mathrm{F}+$ alum in young mice (ANOVA). ${ }^{\#}, P<0.05$ comparing $\mathrm{F}$ to $\mathrm{F}+$ alum in aged mice (ANOVA). Each symbol represents an individual mouse. The dotted line represents the limit of detection. (B) Neutralizing antibody titer. Sera from vaccinated mice were tested for RSV neutralizing activity, ${ }^{*} P<0.05$ comparing $F$ to $F+$ alum in young mice (ANOVA). ${ }^{\#}, P<0.05$ comparing $F$ to $F+$ alum in aged mice (ANOVA). **, $P<0.05$ comparing $F$ in young mice to $F$ in aged mice (ANOVA). ${ }^{\#}, P<0.05$ comparing $F+$ alum in young mice to $F+$ alum in aged mice (ANOVA). The dotted line represents the limit of detection. (C) Body weight. Groups of mice $(n=14)$ were unvaccinated or vaccinated twice i.m. and challenged intranasally with $10^{6}$ PFU RSV strain A2 at day 28. Body weights were recorded daily for 4 days. Data are combined from two independent experiments. 
aged mice. We chose the aluminum salts (alum) as an adjuvant to test our hypothesis. Until recently, alum was the only adjuvant licensed for human use in the USA [14]. Alum-adjuvanted RSV vaccines have been evaluated in elderly humans and in mouse models but the adjuvant effects of alum were not consistent. A study published by Falsey et al. showed no adjuvanting effect of alum on RSV F or RSV G protein vaccines in the elderly [15]. Langley et al. reported that an alumcontaining RSV subunit vaccine had decreased immunogenicity compared to non-adjuvanted $\mathrm{F}$ in the elderly [16]. These observations could be the result of: 1) differences in the quality of the $\mathrm{F}$ antigen used; 2) poor suitability of alum as an adjuvant for a booster vaccine in an RSV-seropositive population; or 3) poor performance of the alum-adjuvanted RSV $F$ vaccine in a setting of immunosenescence. Our current study focuses on the third possibility. We sought to evaluate the immunogenicity of a pure, recombinant RSV F protein adjuvanted with alum in the context of immunosenescence by utilizing an aged BALB/c mouse model. The BALB/c mouse model was chosen since this inbred strain is relatively susceptible to RSV infection [17].

We demonstrate that neutralizing anti-RSV antibody titers correlated with protection in young and aged BALB/C mice vaccinated with RSV F protein vaccines. The $F+$ alum formulation protected to a greater degree than nonadjuvanted $\mathrm{F}$ in both young and aged mice. Notably, F antigen alone induced $\mathrm{T}_{\mathrm{H}}$ 2-type responses and eosinophil infiltration in both young and aged mice, and the addition of alum alleviated lung $\mathrm{T}_{\mathrm{H}}$ 2-type cytokine responses and eosinophil infiltration compared to the non-adjuvanted $\mathrm{F}$ group. However, aged mice achieved lower levels of neutralizing antibodies and less protection than young mice given the same dose of vaccine. While $\mathrm{T}_{\mathrm{H}} 2$ responses were lower in aged mice compared to young mice, the decreased neutralizing antibodies and protection suggest that immune responses were generally diminished in the aged mice compared to the young mice. Importantly, alum did not induce inflammatory chemokines or enhanced immunopathologic lesions following $w t$ RSV challenge in the lung airways of aged mice as was observed in young mice.

\section{Results}

\section{Effect of alum on RSV F protein vaccine efficacy in young} and aged mice

In both young and aged mice, live RSV-infected mice were fully protected from challenge (Figure 1A). A dose titration of RSV F protein ranging from $0.03-3 \mu \mathrm{g}$ was previously performed in young mice in our laboratory and demonstrated that partial protection against $w t$ RSV challenge was achieved with a $0.3 \mu \mathrm{g}$ dose of $\mathrm{F}$ (data not shown). Therefore, we chose $0.3 \mu \mathrm{g}$ of RSV F as an immunization dose in this study to evaluate and compare the effects of adjuvanting $\mathrm{F}$ with alum on mediating protection in young and aged mice. Young mice vaccinated with non-adjuvanted $\mathrm{F}$ alone showed partial viral clearance, while no detectable lung viral load was found in the $\mathrm{F}+$ alum vaccinated group or the live RSV group (Figure 1A). In contrast, aged mice receiving nonadjuvanted $\mathrm{F}$ alone were not protected, and aged mice receiving $\mathrm{F}+$ alum showed partial protection (Figure 1A). Notably, F + alum vaccine significantly reduced viral load compared to non-adjuvanted $\mathrm{F}$ vaccine in both young and aged mice.

To determine the immune correlates of protection against challenge following vaccination, we measured the day 27 serum neutralizing antibody titers in young and aged mice (Figure 1B). The neutralizing antibody titers demonstrated a strong inverse correlation with lung viral titers following RSV infection $\left(\mathrm{r}^{2}=-0.837\right)$. In the young mice, complete protection was observed in the $\mathrm{F}+$ alum group, which also showed the highest mean neutralizing antibody titer of $8.0 \log _{2}$ (Figure 1A and $1 B$ ). A neutralizing antibody titer $\geq 5.82 \log _{2}$ in the $\mathrm{F}$ or $\mathrm{F}+$ alum-vaccinated groups appeared to correlate with lung protection (Figure 1A and 1B). While alum enhanced the neutralizing antibody titers in both the young and aged mice, the neutralizing antibody titers in young mice were of a higher magnitude than that observed in aged mice (Figure 1B). There were significant differences in neutralizing antibody titers between the young and aged mice vaccinated with nonadjuvanted $\mathrm{F}$ alone $(* *=P<0.005$, ANOVA) and between the young and aged $\mathrm{F}+$ alum-vaccinated mice (\#\# $=P<0.005$, ANOVA) (Figure 1B). In contrast, low neutralizing antibody titers were detected in the live RSV + RSV challenge mice. This might be due to timing of sampling [18] or alternatively, point to a role for $\mathrm{T}$ cells and/or mucosal antibodies in mediating protection during live RSV infection. We measured body weight as an indicator of RSV illness severity following RSV challenge [19]. Weight loss was detected only in the placebo group in young mice at day 1 following RSV challenge $(P=0.006)$ (Figure $1 C)$. This result indicated that nonadjuvanted $\mathrm{F}$ and $\mathrm{F}+$ alum vaccination followed by $w t$ RSV challenge did not result in exacerbation of illness as measured by weight loss. Taken together, these data support the conclusion that while alum can boost F-specific antibody responses in aged mice, it does not completely overcome the reduced ability of a senescent immune system to respond to an antigen.

\section{Alum-adjuvanted $\mathrm{F}$ protein induces higher RSV F-specific antibody response compared to non-adjuvanted $\mathrm{F}$ protein in young and aged mice}

Next, we measured RSV F-specific antibody responses in serum and lung homogenates. Overall, the mean serum 
and lung IgG titers observed in the young mice were higher than that observed in aged mice (Figure 2). Both young and aged mice immunized with $\mathrm{F}+$ alum had significantly higher serum RSV F-specific IgG antibody titers compared to placebo or $\mathrm{F}$ vaccine groups $(P<$ 0.0001, ANOVA) (Figure 2A). Non-adjuvanted F and F + alum vaccines induced higher levels of RSV F-specific IgG1 compared to IgG2a in young mice (Figure 2B). The data are consistent with previous observations that alum drives a primarily $\mathrm{T}_{\mathrm{H}}$ 2-biased antibody response $[16,20]$. Regardless of the IgG isotype, we observed that the serum RSV F-specific IgG titers correlated with protection in mice vaccinated with RSV F protein. Live RSV infection induced equivalent titers of RSV F-specific
IgG1 and IgG2a in young mice, while in aged mice the IgG subtype titers were near the limit of detection. In addition, we measured both RSV F-specific IgG and IgA antibodies in the lung homogenates; however, only the IgG antibodies were present at detectable levels in all groups and reported here (Figure 2C). RSV F-specific IgA antibodies were detected only in the live RSVinfected group of both young $\left(5.0 \pm 0.8 \log _{2} \mathrm{EC}_{50}\right.$ titer) and aged mice $\left(4.0 \pm 1.2 \log _{2} \mathrm{EC}_{50}\right.$ titer) (data not shown). The RSV F-specific lung IgG antibodies induced by the different vaccine formulations (Figure $2 \mathrm{C}$ ) displayed an inverse correlation with the lung viral titers shown in Figure 1A. Vaccinating with $\mathrm{F}+$ alum resulted in lung IgG antibody titers with the highest magnitude

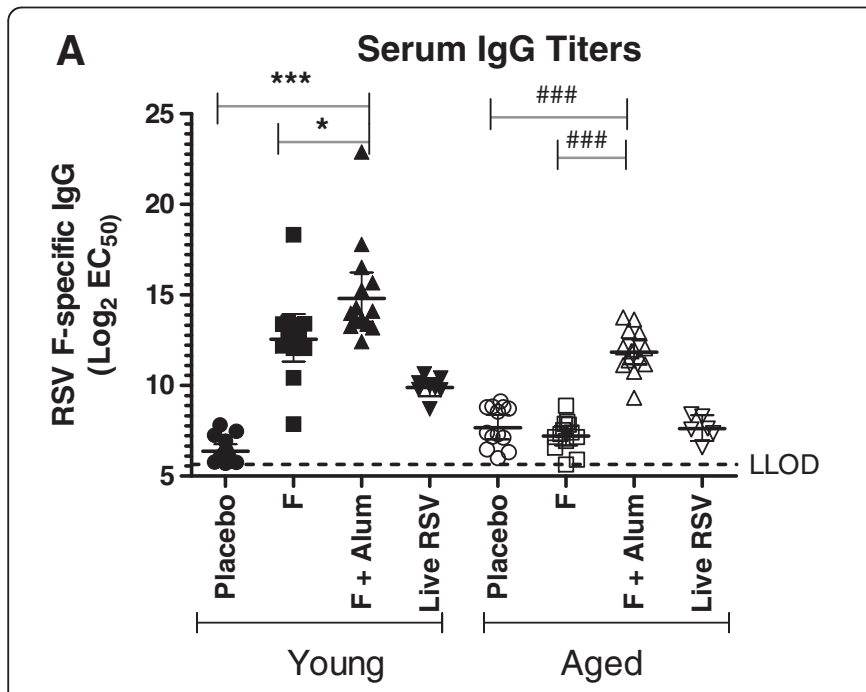

B Serum IgG2a : IgG1
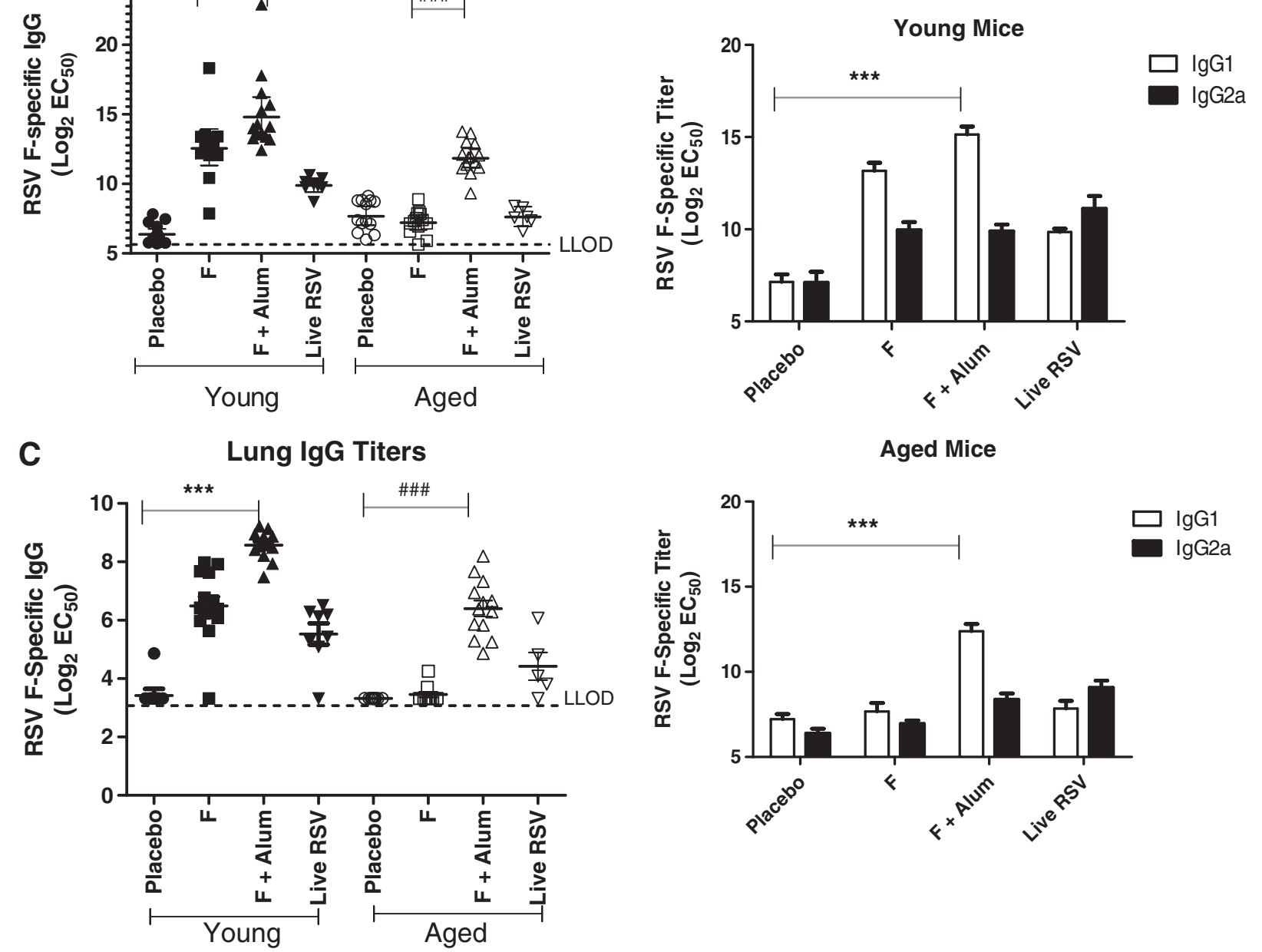

Figure 2 RSV F-specific serum and lung antibody titers in vaccinated young and aged BALB/C mice. Day 27 serum was examined by ELISA for (A) RSV F-binding lgG and (B) RSV F-binding IgG2a or lgG1 subtype antibodies using isotype-specific anti-mouse detection antibodies. (C) Lung homogenates 4 days post-challenge were examined for RSV F-binding IgG antibodies. The dotted line represents the lower limit of detection (LLOD $=1: 50$ dilution). ${ }^{* *}, P<0.0001$ comparing $F+$ alum to placebo in young mice (ANOVA). ${ }^{*}, P<0.05$ comparing $F+$ alum to $F$ in young mice (ANOVA). \#\#\#, $P<0.0001$ comparing $F+$ alum to placebo or $F$ in aged mice (ANOVA). Serology results were combined from two independent experiments. 
compared to the other vaccine groups regardless of the age of the mice (Figure 2C).

\section{Distinct $T_{H}$ 1- and $T_{H}$ 2-type cytokine profiles induced in} the lungs of young and aged mice

To investigate the $\mathrm{T}_{\mathrm{H}} 1$ - and $\mathrm{T}_{\mathrm{H}}$ 2-type immune responses after vaccination and RSV challenge, production of IFN- $\gamma$ ( $\mathrm{T}_{\mathrm{H}} 1$ cytokine) and IL-5 ( $\mathrm{T}_{\mathrm{H}} 2$ cytokine) were measured in lung homogenates (Figure $3 \mathrm{~A}$ and Figure 3B). Overall, significantly lower levels of IFN- $\gamma$ and IL-5 were induced in vaccinated aged mice compared with vaccinated young mice. Interestingly, only live RSV induced IFN- $\gamma$ in both young and aged mice $(\mathrm{P}<0.05)$ (Figure $3 \mathrm{~A})$. High levels of IL-5 were detected in young mice vaccinated with non-adjuvanted $\mathrm{F}$ or $\mathrm{F}+$ alum (Figure 3B). In contrast, we detected basal levels of IL-5 in aged mice (Figure 3B). As IL-5 is implicated in contributing to eosinophilia, we also measured lung eotaxin (CCL11), a chemoattractant that promotes eosinophil recruitment to the lungs [21,22]. The levels of eotaxin in young mice immunized with non-adjuvanted $\mathrm{F}$ were significantly higher than the levels in the other groups (Figure 3C). Relatively low levels of eotaxin were observed in the lungs of all aged mice treatment groups (Figure 3C). The $\mathrm{T}_{\mathrm{H}} 2$ cytokine IL-13, which was also included in our cytokine multiplex panel was detected at low levels $(250 \pm$ $60 \mathrm{pg} / \mathrm{mL}$ ) only in young mice immunized with nonadjuvanted F. IL-13 levels were below the detection limit in aged mice of all treatment groups (data not shown). Levels of the $\mathrm{T}_{\mathrm{H}} 2$ cytokine, IL- 4 , were below the limit of detection of the cytokine multiplex assay (data not shown). Among the chemokines included in our experiments, the only two that were induced to a measurable extent were $\mathrm{KC}$ (CXCL1) and MCP-1 (CCL2) in the non-adjuvanted F vaccinated young mice $(2650 \pm 1250 \mathrm{pg} / \mathrm{mL}$ and $800 \pm 300$ $\mathrm{pg} / \mathrm{mL}$, respectively). In contrast, non-adjuvanted $\mathrm{F}$ vaccinated aged mice did not show a measurable induction of $\mathrm{KC}$ or MCP-1 in the lungs following viral challenge (data not shown).

Differential eosinophil abundance induced in the lungs of young and aged mice following vaccination and wt RSV challenge

We determined histopathologic changes induced by non-adjuvanted $\mathrm{F}$ or $\mathrm{F}+$ alum vaccination followed by $w t$ RSV challenge in young and aged mice. While few eosinophils were observed in placebo and live RSV groups of young and aged mice following challenge, significant eosinophilic infiltrates were observed in young mice vaccinated with non-adjuvanted $\mathrm{F}$ or $\mathrm{F}+$ alum, and in aged mice vaccinated with non-adjuvanted $F$ (Figure 4). The numbers of lung infiltrating eosinophils were significantly lower in aged mice compared with young mice (Figure $4 \mathrm{C}$ ). In addition to the eosinophilic

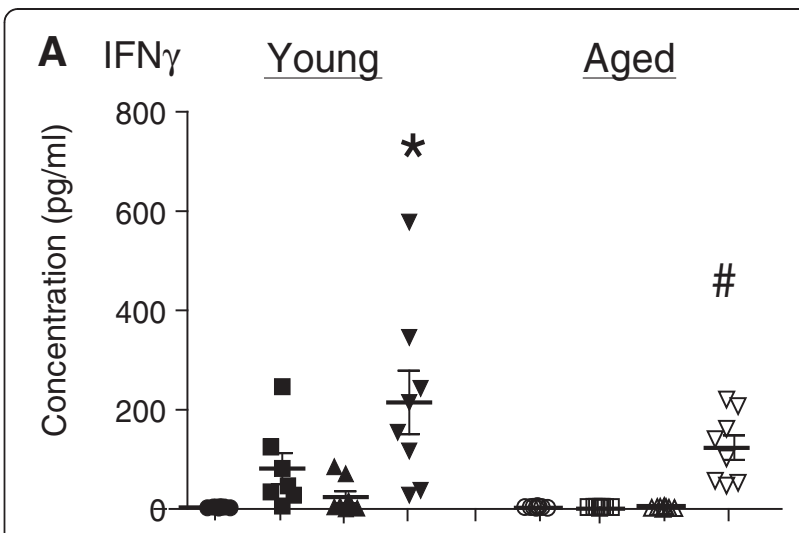

B IL-5
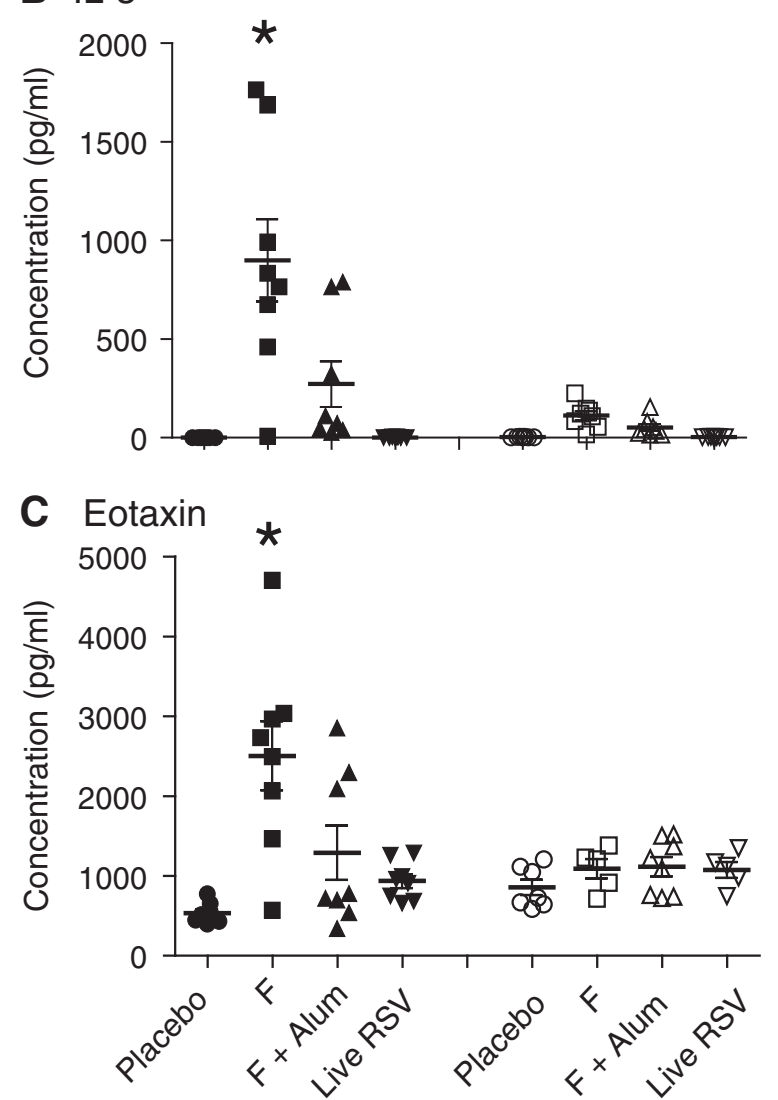

Figure 3 Cytokine levels in the lung homogenates. Cytokines and chemokines in lung homogenates isolated 4 days postchallenge were measured using a 15-plex bead array panel (Luminex). Shown are representative $T_{H} 1$ (IFNY), $T_{H} 2$ (IL-5) cytokines, and eotaxin levels. (A) IFNy levels. ${ }^{*}, P<0.05$ comparing RSV to placebo, $F$, or $F+$ alum in young mice (ANOVA). ${ }^{\#}, P<0.05$ comparing RSV to placebo, $F$, or $F+$ alum in aged mice (ANOVA). (B) IL-5 levels. ${ }^{*}, P<0.05$ comparing $\mathrm{F}$ to $\mathrm{F}+$ alum in young mice (ANOVA). (C) Eotaxin levels. ${ }^{*}, P<0.05$ comparing RSV to placebo, $F$, or $\mathrm{F}+$ alum in young mice (ANOVA). 
infiltrates, we measured other histological features including interstitial pneumonia and perivascular edema in lungs of young and aged mice. However, there were no significant differences in those parameters between the treatment groups (data not shown). Collectively, the score for infiltrating eosinophils in F + alum or nonadjuvanted $\mathrm{F}$ protein vaccinated aged mice was significantly lower compared to the vaccinated young mice following wt RSV challenge (Figure 4C). Further, the eosinophil scores were consistent with the IL-5 and eotaxin levels in the lungs of young and aged mice (Figure 3B and 3C).

\section{Discussion}

Relatively few published studies have delineated the quality or the magnitude of the humoral and lung cytokine response to RSV in a genetically-defined aged host. Zhang et al. demonstrated that aged BALB/c mice have deficient RSV-specific $\mathrm{CD}^{+}$cytolytic $\mathrm{T}$ cell responses and IFN $\gamma$ production, but RSV antigen-specific humoral responses, histopathologic changes, and viral loads were not evaluated in this study [23].

Purified RSV fusion (F), attachment (G), and matrix (M) proteins have been developed as subunit vaccines; however, RSV subunit vaccines have been plagued with poor immunogenicity in the elderly [6-11]. These studies indicated a need for investigating the ability of a highly pure RSV F preparation to induce immune responses in a genetically defined aged host. Based on these and other similar findings in the field we decided to explore if an adjuvant-driven, respiratory virus antigen-specific immune response could alleviate or correct the immune defects observed in the lungs of aged mice. In order to address the above question, we expressed a recombinant RSV F protein in mammalian cells and purified it to $>$ 95\% purity using immuno-affinity methods. Properties

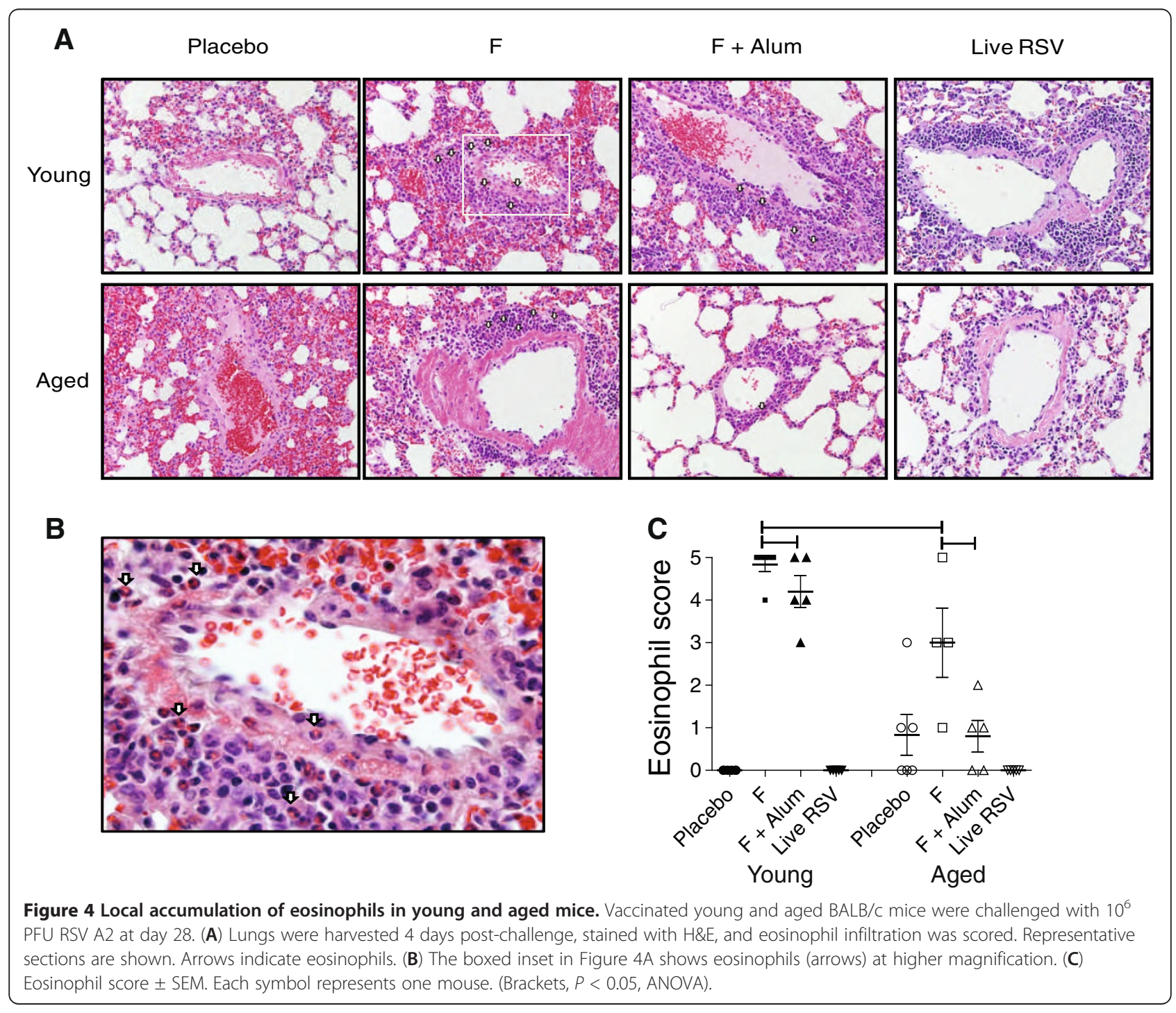


such as protein folding, aggregation and integrity of the antigenic sites in the purified RSV F protein were carefully characterized using a variety of biophysical and biochemical techniques that included ELISA, SDS-PAGE, Western blotting and electron microscopy. Furthermore, the alum-adjuvanted RSV F formulation was characterized using ELISA and Western blotting to ensure the preservation of the RSV F antigenic sites.

Recently published work has elucidated the possible in vivo mechanism by which alum mediates its adjuvant activity [24]. The authors demonstrated that in mice, alum causes cell death and the subsequent release of host cell DNA acts as a potent endogenous immunostimulatory signal mediating the induction of IgG1 and $\mathrm{T}_{\mathrm{H}} 2$ responses [24]. The induction of serum IgG1 antibodies by alum observed in our studies (Figure 2B) may be attributable to this damage-associated molecular pattern recognition of the host cell DNA, mediating adjuvant activity on humoral immune responses. Another study describing the potential molecular mechanism of alum activity demonstrated that alum directly interacts with dendritic cell membrane lipids, triggering signaling cascades that promote $\mathrm{CD}_{4}^{+} \mathrm{T}$ cell activation and humoral immune responses [25].

In this study, we evaluated the effect of alum adjuvant on host immune responses against RSV F protein in an RSV seronegative immunosenescent setting. Both serum neutralizing antibody titers and lung cytokine production in response to vaccination and $w t$ RSV challenge were found to be diminished in aged mice compared to young mice (Figure 1 and Figure 3 ). However, lung eosinophilic inflammation following vaccination and challenge was also lower in aged mice compared to young mice, and $\mathrm{F}+$ alum vaccination did not induce additional immunopathologic lesions in aged mice relative to the placebo group (Figure 4). The relatively low eosinophilic infiltrates observed in the placebo and live RSV-infected groups (Figure 4) supported the notion that lung immunopathology is caused by the host's inflammatory immune response to viral replication rather than being a direct response to the viral load found in the lungs following $w t$ RSV challenge [26].

Our findings from the studies presented here were consistent with the lack of evidence for vaccine-mediated disease enhancement in this model reflecting the low risk of inducing a severe inflammatory response to alum immunization in aged hosts. Despite lower immune responses, $\mathrm{F}+$ alum vaccinated aged mice produced significant neutralizing antibodies to RSV and achieved a 2log reduction in lung viral titers compared to the nonadjuvanted F cohort (Figure 1). Significant RSV F-specific IgG titers were measured in the lung homogenates of the $\mathrm{F}+$ alum and live RSV infection groups (Figure 2C); however, the individual contributions of serum antibodies present in the blood versus those secreted by lung plasma cells could not be distinguished by our methods. Alumadjuvanted RSV F protein reduced lung viral titers by 2$\log$ in aged mice, though the protection conferred was suboptimal compared to that seen in young mice (3-log reduction in viral titer) (Figure 1). Taken together, our data suggest that a higher $\mathrm{F}$ antigen dose and/or a stronger adjuvant may be required in immunosenescent populations to attain an appropriate balance of protective neutralizing antibodies and effective $\mathrm{T}_{\mathrm{H}} 1$-dominant cytokine responses along with minimal lung immunopathology.

Our model evaluated humoral and cytokine responses induced by $w t$ RSV A2 intranasal infection and boosted by challenge. Interestingly, this group was the only one among all of the treatment groups that generated statistically significant lung $\mathrm{T}_{\mathrm{H}} 1$ cytokine responses (Figure 3). Additionally, RSV F-specific IgA antibodies were only detected in the lung homogenates of the live RSV-infected animals, pointing to a role for mucosal antibodies in mediating protection against natural RSV infection in both young and aged mice. The low serum neutralizing antibody titers measured in the live RSV-infected groups (Figure 1B) further supported our conclusion that protection against viral challenge was mediated by RSV Fspecific IgA antibodies and/or IFN- $\gamma$ responses induced in the lungs of the animals in this group. The relationship between the elicited mucosal antibody response at the local site of infection and protection against viral challenge in this group may have important implications for RSV disease in the elderly and deserves further investigation. However, the detailed evaluation of a vaccine-specific immune response in a RSV-seropositive, aged mouse model as it relates to vaccine responses induced in elderly humans was outside the scope of this study. The characterization of immune responses in a RSVseropositive vaccinated aged mouse model is expected to provide additional mechanistic insight, facilitating the rational design of enhanced immunization strategies against RSV disease in the elderly.

\section{Conclusions}

In this study, we found that alum-adjuvanted RSV F protein was protective in aged mice, though the protection was sub-optimal compared to that seen in young mice. Overall, day 27 serum neutralizing antibody titers and lung cytokines produced in response to vaccination and RSV challenge were diminished in aged mice compared to young mice. However, $\mathrm{F}+$ alum vaccination did not induce additional immunopathology in aged mice relative to vaccination with unadjuvanted $\mathrm{F}$ protein following RSV challenge. Despite lower immune responses, $\mathrm{F}+$ alum vaccinated aged mice produced significant neutralizing antibodies against RSV and achieved a 2-log reduction in lung viral titers compared to the unadjuvanted F cohort. Taken 
together, these data suggest that a higher $\mathrm{F}$ antigen dose and/or a stronger adjuvant may be required in immunosenescent populations to attain an appropriate balance of protective neutralizing antibodies, effective $\mathrm{T}_{\mathrm{H}}$ 1-biased cytokine response and minimal lung immunopathology.

\section{Methods \\ Mice}

Pathogen-free, 8-week- and 18-month-old, female $\mathrm{BALB} / \mathrm{c}$ mice were purchased from the National Institute of Aging (NIA, Bethesda, MD). All animal procedures were conducted according to the guidelines of the Institutional Animal Care and Use Committee.

\section{RSV antigens}

The fusion subunit $\mathrm{F}$ protein (sequence containing amino acids 1-524) of RSV A2 was expressed in Chinese Hamster Ovary (CHO) cells (ATCC, Manassas, VA) and immuno-affinity purified with anti-RSV $\mathrm{F}$ monoclonal antibody (Palivizumab, MedImmune, Gaithersburg, MD) to $>95 \%$ purity. The antigenic sites of the protein were preserved as determined by an ELISA sandwich assay. Purified RSV F protein was used for both animal immunizations and coating in ELISA assays. Our data suggested that any anti-CHO host cell protein response generated in young or aged mice was not detectable in an ELISA assay using $0.5 \mu \mathrm{g} / \mathrm{mL}$ coating concentration of RSV F. Wildtype $(w t)$ RSV A2 and Green Fluorescent Protein (GFP)tagged RSV A2 were grown and titrated in Vero cells. Virus stocks were stabilized in sucrose-phosphate (1X SP) buffer $\left(0.2 \mathrm{M}\right.$ sucrose, $0.0038 \mathrm{M} \mathrm{KH_{2 }} \mathrm{PO}_{4}, 0.0072 \mathrm{M}$ $\mathrm{KH}_{2} \mathrm{PO}_{4}$ ), snap-frozen and stored at $-70^{\circ} \mathrm{C}$.

\section{Study design}

All mice were primed at day 0 and boosted at day 14 intramuscularly (i.m) in both quadriceps. Placebo groups were given $100 \mu \mathrm{l}$ of phosphate buffered saline (PBS), while $\mathrm{F}$ vaccine groups received $0.3 \mu \mathrm{g}$ of $\mathrm{F}$ protein. This dose was based on our previous observation that $0.3 \mu \mathrm{g}$ of $\mathrm{F}$ without adjuvant was only partially protective upon $w t$ RSV A2 challenge (unpublished results). For RSV F + alum group, mice were given $0.3 \mu \mathrm{g}$ of $\mathrm{F}$ protein mixed (by vortexing for $30 \mathrm{~min}$ at RT) with $100 \mu \mathrm{g}$ of Alhydrogel (Aluminum Hydroxide, Brenntag Biosector, Denmark). Lastly, for the live RSV group, mice were intranasally (i.n.) infected on day 0 with $10^{6}$ PFU wt RSV strain A2. Protection was assessed by intranasal inoculation of all mice with $10^{6}$ PFU RSV strain A2 on day 28. Body weight was measured daily following wt RSV challenge until animals were euthanized.

\section{Serum collection}

Peripheral blood was collected from the submandibular vein 27 days post-priming. Collected blood was left at room temperature (RT) for $20 \mathrm{~min}$. The tubes were centrifuged at $7000 \mathrm{rpm}$ for $10 \mathrm{~min}$ and sera stored at $-80^{\circ} \mathrm{C}$.

\section{Micro-neutralization assay}

RSV-specific neutralizing antibody titers in mouse sera were measured using GFP-expressing RSV A2 in a micro-neutralization assay. Mouse sera from all treatment and placebo groups were heat-inactivated at $56^{\circ} \mathrm{C}$ for $45 \mathrm{~min}$ and serially diluted three-fold in growth medium. Equal volumes of the diluted sera were mixed with GFP-expressing RSV A2 virus to yield 500 PFU/ well. Virus only and hyperimmune serum with a known neutralizing antibody titer were included on each plate as controls. The serum + virus (or virus only) mixture was incubated at $33^{\circ} \mathrm{C}, 5 \% \mathrm{CO}_{2}$ for $1 \mathrm{~h}$ to allow time for the neutralizing antibodies in the serum to neutralize the virus particles. Confluent monolayers of Vero cells (ATCC, Manassas, VA) prepared in separate 96-well plates were infected with the GFP-expressing RSV A2 virus only or the serum/virus mixture from the above step. The infected Vero cell plates were incubated at $33^{\circ} \mathrm{C}$, $5 \% \mathrm{CO}_{2}$ for $22 \mathrm{~h}$. The plates were washed and the fluorescent viral foci enumerated using an IsoCyteTM Reader (Blueshift Biotechnologies, Sunnyvale, CA). The 50\% reduction in viral foci (antibody $\mathrm{EC}_{50}$ titers) was calculated using a 4-parameter curve fit algorithm.

\section{Serum IgG, IgG1, IgG2a, and Lung IgG ELISA}

RSV F-specific IgG antibody titers in serum were measured using ELISA. Three sets of high binding 96-well plates intended to measure IgG, IgG1, and IgG2a, respectively, were coated overnight at $4^{\circ} \mathrm{C}$ with $0.5 \mu \mathrm{g} / \mathrm{mL}$ of $\mathrm{CHO}$-expressed recombinant RSV F diluted in PBS. Plates were washed with PBS/0.05\% Tween-20 and blocked with PBS/0.05\% Tween-20 containing $0.5 \%$ bovine serum albumin (BSA, EMD Biosciences, Gibbstown, $\mathrm{NJ}$ ) for $1 \mathrm{~h}$ at $37^{\circ} \mathrm{C}$. The samples and hyperimmune serum reference standard (MedImmune, Mountain View, CA) were serially diluted 2-fold in PBS/0.05\% Tween-20/0.5\% BSA following an initial pre-dilution of 1:50. After washing, the pre-diluted samples were added to the three plates and the plates incubated at $37^{\circ} \mathrm{C}$ for 1 hour. Horseradish peroxidase (HRP)-conjugated, goat anti-mouse IgG, IgG1, or IgG2a antibodies (Jackson ImmunoResearch, West Grove, PA) diluted 1:20,000 in PBS/0.05\% Tween-20/0.5\% BSA were added, respectively, to the three plates. After washing, the plates were then developed with 3,3',5,5'-tetramethylbenzidine (TMB, Sigma, St. Louis, MO) and stopped with $1 \mathrm{~N} \mathrm{HCl}$ (Sigma, St. Louis, MO). The absorbance was measured at $450 \mathrm{~nm}$ on a SpectraMax plate reader and analyzed using SoftMax ${ }^{\circledR}$ Pro (Molecular Devices Inc., Sunnyvale, $\mathrm{CA})$. Titers were reported as $\log _{2} \mathrm{EC}_{50}$ determined using a 4-parameter curve fit for each sample curve. 


\section{Viral plaque assay}

Mice were euthanized at day 4 post-challenge. A Beadbeater (Biospec Products, Bartlesville, OK) was used to homogenize the lungs as previously described [27]. Lung homogenates were serially diluted and used to inoculate subconfluent HEp-2 cells in 24-well plates. After $1 \mathrm{~h}$ adsorption at RT on a rocking platform, the cells were overlaid with $\mathrm{MEM} / 10 \% \mathrm{FBS} / 1 \%$ penicillin G/streptomycin sulfate/amphotericin B solution $/ 0.75 \%$ methylcellulose. After six days, the overlay medium was removed and the cells fixed with methanol. Plaques were visualized by immunodetection as described [26].

\section{Cytokine quantification}

Lung homogenates prepared for the viral plaque assay as described above were also evaluated in a Luminex-based cytokine profiling assay. Mouse cytokine multiplex kits, custom designed to include IL-5, IL-13, IFN- $\gamma$, RANTES, MCP-1, eotaxin, KC, IP-10, and MIP- $1 \alpha$ were purchased from Millipore (Billerica, MA). The assay was performed according to the manufacturer's instructions. The plates were analyzed on a Bio-Rad Luminex reader (Hercules, $\mathrm{CA})$, and individual cytokine levels were expressed as $\mathrm{pg} / \mathrm{mL}$. IFN $\gamma$ and IL-5 were used to represent $\mathrm{T}_{\mathrm{H}} 1$ and $\mathrm{T}_{\mathrm{H}} 2$ cytokines, respectively.

\section{Lung histopathology}

Heart-lung blocks were harvested 4 days post-infection and fixed overnight in $4 \%$ paraformaldehyde. Lungs were transferred to $70 \%$ ethanol and then embedded in paraffin blocks as described previously [27]. Tissue sections $(5 \mu \mathrm{m})$ were stained with hematoxylin and eosin $(\mathrm{H} \& \mathrm{E})$ to assess histologic changes. H\&E-stained slides were digitally scanned using a Zeiss MIRAX MIDI microscope [27]. Slides were examined and scored by a pathologist who was blinded to the experimental groups. Lymphocytes, neutrophils, macrophages, and eosinophils were assessed in peribronchiolar, perivascular, interstitial, and alveolar spaces as described [27]. For the eosinophil score, groups were assessed for severity of eosinophilic infiltrate on a scale of 0 to 5 in the peribronchiolar, perivascular, interstitial, and alveolar spaces, where $0=$ no eosinophils present, $1=1-10$ in area, $2=11-20,3=21-30$, $4=31-40,5=41-50$.

\section{Abbreviations \\ RSV: Respiratory Syncytial Virus; Alum: Aluminum Hydroxide; GFP: Green Fluorescent Protein; TMB: 3,3',5,5'-Tetramethylbenzidine.}

\section{Competing interests}

The authors declare that they have no competing interests.

\section{Author's contributions}

A.C, S.L (Stacie Lambert), E.S, M.M and S.L (Sujin Lee) designed the described studies; A.C, K.S (Kate Stokes), K.P, H.K. and S. L (Sujin Lee) performed the described experiments; K.S (Kaori Sakamoto) performed hispathology. All authors interpreted the described data and contributed to the writing of the manuscript. All authors read and approved the final manuscript.

\section{Acknowledgements}

We wish to thank Dr. Li Yu for performing the correlation statistical analysis for these studies. We also wish to thank Dr. Roderick Tang, Dr. Ed Mocarski and Dr. Gary Van Nest for their input on the study designs and critical review of the results.

\section{Funding}

This work was sponsored by Medlmmune, LLC. This work was supported by $\mathrm{NIH}$ grants 1R01Al087798 and 1U19AI095227 (M. Moore) as well as Emory Egleston Children Research Center (EECRC) grant and American Federation for Aging Research (AFAR) grant (Sujin Lee).

\section{Author details}

'Infectious Diseases/Vaccines Research, Medlmmune, LLC, Mountain View, CA, USA. ${ }^{2}$ Department of Pediatrics, Emory Children's Center, Emory University, Atlanta, GA, USA. ${ }^{3}$ Children's Healthcare of Atlanta, Atlanta, GA, USA. ${ }^{4}$ Department of Pathology, College of Veterinary Medicine, University of Georgia, Athens, GA, USA.

Received: 8 August 2012 Accepted: 28 September 2012

Published: 2 October 2012

\section{References}

1. Falsey AR, Hennessey PA, Formica MA, Cox C, Walsh EE: Respiratory syncytial virus infection in elderly and high-risk adults. N Engl J Med 2005, 352(17):1749-1759.

2. Glezen WP, Taber LH, Frank AL, Kasel JA: Risk of primary infection and reinfection with respiratory syncytial virus. Am J Dis Child 1986, 140(6):543-546.

3. Falsey AR, Walsh EE: Respiratory syncytial virus infection in elderly adults. Drugs Aging 2005, 22(7):577-587.

4. Elliot AJ, Fleming DM: Influenza and respiratory syncytial virus in the elderly. Expert Rev Vaccines 2008, 7(2):249-258.

5. Graham BS: Biological challenges and technological opportunities for respiratory syncytial virus vaccine development. Immunol Rev 2011, 239(1):149-166.

6. Neuzil KM, Johnson JE, Tang YW, Prieels JP, Slaoui M, Gar N, Graham BS: Adjuvants influence the quantitative and qualitative immune response in $B A L B / c$ mice immunized with respiratory syncytial virus FG subunit vaccine. Vaccine 1997, 15(5):525-532.

7. Welliver RC, Tristram DA, Batt K, Sun M, Hogerman D, Hildreth S: Respiratory syncytial virus-specific cell-mediated immune responses after vaccination with a purified fusion protein subunit vaccine. J Infect Dis 1994, 170(2):425-428.

8. Falsey AR, Walsh EE: Safety and immunogenicity of a respiratory syncytial virus subunit vaccine (PFP-2) in the institutionalized elderly. Vaccine 1997, 15(10):1130-1132.

9. Falsey AR, Walsh EE: Safety and immunogenicity of a respiratory syncytial virus subunit vaccine (PFP-2) in ambulatory adults over age 60. Vaccine 1996, 14(13):1214-1218.

10. Murata Y: Respiratory syncytial virus vaccine development. Clin Lab Med 2009, 29(4):725-739.

11. Empey KM, Peebles RS Jr, Kolls JK: Pharmacologic advances in the treatment and prevention of respiratory syncytial virus. Clin Infect Dis 2010, 50(9):1258-1267.

12. Murphy BR, Sotnikov AV, Lawrence LA, Banks SM, Prince GA: Enhanced pulmonary histopathology is observed in cotton rats immunized with formalin-inactivated respiratory syncytial virus (RSV) or purified $\mathrm{F}$ glycoprotein and challenged with RSV 3-6 months after immunization. Vaccine 1990, 8(5):497-502.

13. Vaux-Peretz F, Chapsal JM, Meignier B: Comparison of the ability of formalin-inactivated respiratory syncytial virus, immunopurified $F, G$ and $\mathrm{N}$ proteins and cell lysate to enhance pulmonary changes in Balb/c mice. Vaccine 1992, 10(2):113-118.

14. Baylor NW, Egan W, Richman P: Aluminum salts in vaccines-US perspective. Vaccine 2002, 20(Suppl 3):S18-S23.

15. Falsey AR, Walsh EE, Capellan J, Gravenstein S, Zambon M, Yau E, Gorse GJ, Edelman R, Hayden FG, McElhaney JE, et al: Comparison of the 
safety and immunogenicity of 2 respiratory syncytial virus (rsv) vaccines-nonadjuvanted vaccine or vaccine adjuvanted with alumgiven concomitantly with influenza vaccine to high-risk elderly individuals. J Infect Dis 2008, 198(9):1317-1326.

16. Langley JM, Sales V, McGeer A, Guasparini R, Predy G, Meekison W, Li M, Capellan J, Wang E: A dose-ranging study of a subunit Respiratory Syncytial Virus subtype $A$ vaccine with and without aluminum phosphate adjuvantation in adults $>$ or $=65$ years of age. Vaccine 2009, 27(42):5913-5919.

17. Prince GA, Horswood RL, Berndt J, Suffin SC, Chanock RM: Respiratory syncytial virus infection in inbred mice. Infect Immun 1979, 26(2):764-766.

18. Hancock GE, Hahn DJ, Speelman DJ, Hildreth SW, Pillai S, McQueen K: The pulmonary immune response of Balb/c mice vaccinated with the fusion protein of respiratory syncytial virus. Vaccine 1994, 12(3):267-274.

19. Graham BS, Bunton LA, Wright PF, Karzon DT: Role of T lymphocyte subsets in the pathogenesis of primary infection and rechallenge with respiratory syncytial virus in mice. J Clin Invest 1991, 88(3):1026-1033.

20. Romanowski B, Schwarz TF, Ferguson LM, Peters K, Dionne M, Schulze K, Ramjattan B, Hillemanns P, Catteau G, Dobbelaere K, et al: Immunogenicity and safety of the HPV-16/18 AS04-adjuvanted vaccine administered as a 2-dose schedule compared with the licensed 3-dose schedule: results from a randomized study. Hum Vaccin 2011, 7(12):1374-1386.

21. Collins PD, Marleau S, Griffiths-Johnson DA, Jose PJ, Williams TJ: Cooperation between interleukin- 5 and the chemokine eotaxin to induce eosinophil accumulation in vivo. J Exp Med 1995, 182(4):1169-1174.

22. Kariyawasam HH, Robinson DS: The eosinophil: the cell and its weapons, the cytokines, its locations. Semin Respir Crit Care Med 2006, 27(2):117-127.

23. Zhang $Y$, Wang $Y$, Gilmore $X$, Xu K, Wyde PR, Mbawuike IN: An aged mouse model for RSV infection and diminished CD8(+) CTL responses. Exp Biol Med (Maywood) 2002, 227(2):133-140.

24. Marichal T, Ohata K, Bedoret D, Mesnil C, Sabatel C, Kobiyama K, Lekeux P, Coban C, Akira S, Ishii K, et al: DNA released from dying host cells mediates aluminum adjuvant activity. Nat Med 2011, 17(8):996-1002.

25. Flach $T L, N g$ G, Hari A, Desrosiers MD, Zhang P, Ward SM, Seamone ME, Vilaysane A, Mucsi AD, Fong $Y$, et al: Alum interaction with dendritic cell membrane lipids is essential for its adjuvanticity. Nat Med 2011, 17(4):479-487.

26. Moore ML, Chi MH, Luongo C, Lukacs NW, Polosukhin W, Huckabee MM, Newcomb DC, Buchholz UJ, Crowe JE Jr, Goleniewska K, et al: A chimeric A2 strain of respiratory syncytial virus (RSV) with the fusion protein of RSV strain line 19 exhibits enhanced viral load, mucus, and airway dysfunction. J Virol 2009, 83(9):4185-4194.

27. Stokes KL, Chi MH, Sakamoto K, Newcomb DC, Currier MG, Huckabee MM, Lee S, Goleniewska K, Pretto C, Williams JV, et al: Differential pathogenesis of respiratory syncytial virus clinical isolates in BALB/C mice. J Virol 2011 85(12):5782-5793.

doi:10.1186/1742-4933-9-21

Cite this article as: Cherukuri et al:: An adjuvanted respiratory syncytial virus fusion protein induces protection in aged BALB/c mice. Immunity \& Ageing 2012 9:21.

\section{Submit your next manuscript to BioMed Central and take full advantage of:}

- Convenient online submission

- Thorough peer review

- No space constraints or color figure charges

- Immediate publication on acceptance

- Inclusion in PubMed, CAS, Scopus and Google Scholar

- Research which is freely available for redistribution

Submit your manuscript at www.biomedcentral.com/submit
C BioMed Central 